\title{
Call for New Model Systems for Studying Psychiatric Diseases
}

\section{Xiaomeng Han ${ }^{1 *}$ and Kan Xiong ${ }^{2,3}$}

${ }^{1}$ Deparment of Neurobiology, Harvard Medical School, Boston, MA 02215, USA

${ }^{2}$ Broad Institute of MIT and Harvard, Cambridge, MA, 02142, USA

${ }^{3}$ Department of Biological Engineering, MIT, Cambridge, MA, 02142, USA

Psychiatric diseases make individual patients suffer, and represent major problems in human society. Over the past few decades, human society has witnessed amazing breakthroughs in treatments for infectious diseases, metabolic disorders and malignant cancers. However, there have been very minor advances in treating mental diseases which make individual patients suffer, and now represent major problems in our society. For example, since the 1950s, new molecules approved by the FDA for treating anxiety disorder have been limited to benzodiazepines, atypical anxiolytics buspirone, meprobamate and hydroxyzine pamoate [1]. This is due to the fact that our understanding of mental illnesses has been very poor. One major hurdle for studying psychiatric diseases is that we do not have a good organism(s) for modeling complex psychiatric diseases of human beings.

The challenge in modeling psychiatric diseases lies in the fact that insofar, there has been no reliable lab test for mental illnesses. We cannot tell by blood test or ultrasound that someone's depression has recovered or not. Mental illnesses' diagnosis and evaluation are mainly based on psychiatrists' conversation with patients. Therefore, it's nearly impossible to accurately model one disease with an animal which cannot talk, not to mention the vast difference between the physiology of humans and animals.

Fortunately, recent years have witnessed several major progresses in the modeling of mental illnesses. First of all, thanks to the progress in the field of stem cell research, it is now possible to take a patient's skin cells and reprogram them into induced pluripotent stem cells (iPSCs) [2]. What's more, it is feasible to use iPSCs to grow cerebral organoids in a dish. With correct conditions, iPSCs can be induced into neuroectodermal progenitor cells, and then be coaxed into three dimensional structures which resemble the stratified human cerebral cortex [3]. Recently, using the most refined lineage reprogramming protocol, scientists can even change individual neurons' fate and control the type of neurons that a progenitor cell will differentiate into [4]. We can imagine, by using these newly developed in vitro models, it will be feasible to take fibroblasts from patients who suffer from neurodevelopmental diseases like schizophrenia and then differentiate them into cerebral organoids for studying the genetic mechanisms of these devastating diseases and probably use these models to screen for new compounds which could potentially lead to new therapies.

What's more, the most popular genomic editing tool nowadays, the CRISPR/ Cas9 system [5] will also help develop models for mental illnesses. In the past, the generation of genetically modified organisms for studying psychiatric diseases has long been limited by genomic editing tools' low efficiency, poor specificity and high cost. The new CRISPR/Cas9 system has transformed our ability to edit the genomes of model organisms [6], making it possible for us to generate more desirable animal lines or cell lines in a relatively short time period and at a relatively low cost. Given CRISPR/Cas9 system's capability for efficient and simplified tailoring of the mouse genome [7], engineering humanized mice will be feasible. These humanized mouse models can not only be used for studying the functions of genes in diseases identified by genome-wide association studies (GWAS) [8], but also complement with various behavior tests to deepen our understanding of psychiatric diseases.

Last but not the least, there is a new kind of primate which can be potentially used as a better model organism for studying mental illnesses. Although mice have been widely used as model organisms, they are still rodents, which greatly limits their usage in modeling the higher level cognitive functions of human beings. Monkeys are better, but they are difficult to breed and handle. Recently, more and more researchers have begun to use marmosets (Callithrix) for modeling psychiatric diseases [9]. Marmosets are relatively small compared to monkeys, and their reproductive cycle is shorter. They also form monogamous, biparental social relationships and frequently give birth to twins. These features make marmoset an attractive candidate for easy gene manipulation and for studying gene-environment interaction [10]. These days, some researchers are also using CRISPR/Cas9 to construct gene-engineered marmoset lines for studying psychiatric diseases [11].

After all, with recent advancements, we will be able to develop better organism models for studying mental illnesses. It is no hard to imagine that in the next decade, there will be many breakthroughs in our knowledge of psychiatric diseases which will facilitate the development of new treatments for mental illnesses.

\section{References}

1. Kinch MS, Haynesworth A, Kinch SL, Hoyer D (2014) An overview of FDAapproved new molecular entities: 1827-2013. Drug Discov Today 19: 10331039.

2. Takahashi K, Tanabe K, Ohnuki M, Narita M, Ichisaka T, et al. (2007) Induction of pluripotent stem cells from adult human fibroblasts by defined factors. Cell 131: 861-872.

3. Sasai $Y(2013)$ Next-generation regenerative medicine: organogenesis from stem cells in 3D culture. Cell Stem Cell 12: 520-530.

4. Ye Z, Mostajo-Radji MA, Brown JR, Rouaux C, Tomassy GS, et al. (2015) Instructing Perisomatic Inhibition by Direct Lineage Reprogramming of Neocortical Projection Neurons. Neuron 88: 475-483.

5. Cong L, Ran FA, Cox D, Lin S, Barretto R, et al. (2013) Multiplex Genome Engineering Using CRISPR/Cas Systems. Science 339: 819-823

6. Mali P, Esvelt KM and Church GM (2013) Cas9 as a versatile tool for engineering biology. Nature Methods 10: 957-963.

7. Deussing JM (2013) Targeted mutagenesis tools for modelling psychiatric disorders. Cell Tissue Res 354: 9-25.

*Corresponding author: Xiaomeng Han, Deparment of Neurobiology, Harvard Medical School, Boston, MA 02215, USA, Tel: +1-857-293-3162; E-mail: xiaomenghan@g.harvard.edu

Received December 20, 2015; Accepted December 24, 2015; Published January 07, 2016

Citation: Han X, Xiong X (2016) Call for New Model Systems for Studying Psychiatric Diseases. J Biomol Res Ther 5: e142. doi:10.4172/2167-7956.1000e142

Copyright: (c) 2016 Han X, et al. This is an open-access article distributed unde the terms of the Creative Commons Attribution License, which permits unrestricted use, distribution, and reproduction in any medium, provided the original author and source are credited. 
Citation: Han X, Xiong X (2016) Call for New Model Systems for Studying Psychiatric Diseases. J Biomol Res Ther 5: e142. doi:10.4172/2167$7956.1000 \mathrm{e} 142$

Page 2 of 2

8. Visscher PM, Brown MA, McCarthy MI, Yang J (2012) Five years of GWAS discovery. Am J Hum Genet 90: 7-24.

9. Pryce CR, Dettling AC, Spengler M, Schnell CR, Feldon J (2004) Deprivation of parenting disrupts development of homeostatic and reward systems in marmoset monkey offspring. Biol Psychiatry 56: 72-79.
10. Nelson EE and Winslow JT (2009) Non-human primates: model animals for developmental psychopathology. Neuropsychopharmacology 34: 90-105.

11. Kumita W, Sato KY, Shimada A, Oiwa R, Kuge T, et al. (2014) Attempts to generate knock in marmosets using the CRISPR/Cas9 system. Transgenic Research 23: 874-874. 